A. Pramesh Rao, G. Swarup and Gopal-Krishna, eds.

\title{
Recombination Radio Lines at Very Low Frequencies
}

\author{
A. A. Konovalenko, S. V. Stepkin and D. V. Shalunov \\ Institute of Radio Astronomy, National Academy of Sciences of \\ Ukraine, \& Chervonopraporna St., 61002, Kharkov, Ukraine
}

\begin{abstract}
Investigations of carbon low frequency radio recombination lines measured with the world biggest decameter wavelength array UTR-2 are described.
\end{abstract}

Carbon radio recombination lines (RRL) at low frequencies are convenient means for interstellar medium diagnostics (Konovalenko 1990) . Decameter radio telescope UTR-2 provides the possibility to observe them at the lowest frequencies. During the last years new results were obtained with this telescope and 128-channel digital correlometer (Stepkin 1996).

Fig. 1 shows the high accuracy spectrum of carbon RRL near $26 \mathrm{MHz}$ $(n \sim 630)$ towards Cas A (integration time is near $700 \mathrm{~h}$ ). New methods of Voigt profile fitting are used. Obtained data correspond to the model with line formation in a diffused non-homogeneous and comparatively warm partially ionized gas, including dielectronic-like recombination process. New observations show absorption lines outside of Cas A limb (up to few degrees from its center). It confirms the presence of distributed CII regions in this direction.

The most low frequency spectral lines were detected near $12 \mathrm{MHz}, n \sim 812$ (Fig. 2a). Their width $\Delta \nu_{L}$ is increased dramatically as we can see from Fig. $2 \mathrm{~b}\left(\Delta \nu_{D}\right.$ is Doppler broadening, $\Delta \nu_{R}$ is radiative broadening).

Fig. 3 shows the detection of the biggest principal quantum number (upto 868 ) by the observations of $\beta$-lines near $20 \mathrm{MHz}$. Such strong excited atoms are very sensitive to the physical conditions in the low density interstellar plasma.

Progress of these investigations is connected to improvement of sensitivity. This is possible when a series of such lines is observed simultaneously (for example, in frequency range $20-30 \mathrm{MHz}$ almost $100 \alpha$-lines and few hundreds $\beta$-lines could be investigated). Modern digital techniques can solve this task. It is important to investigate this phenomenon in the Galaxy at very low $(\nu<30$ $\mathrm{MHz})$ and low $(\nu \sim 300 \mathrm{MHz})$ frequencies.

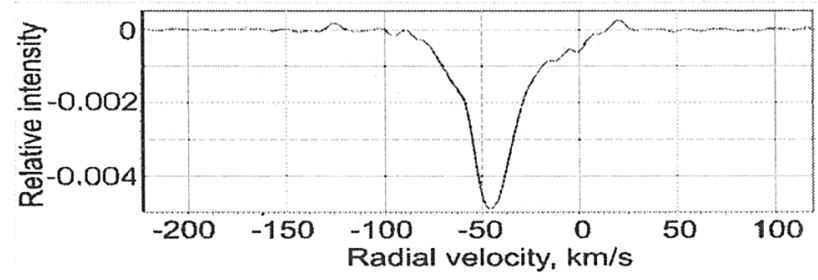

Figure 1. Carbon RRL near $26 \mathrm{MHz}$ towards Cas A. 

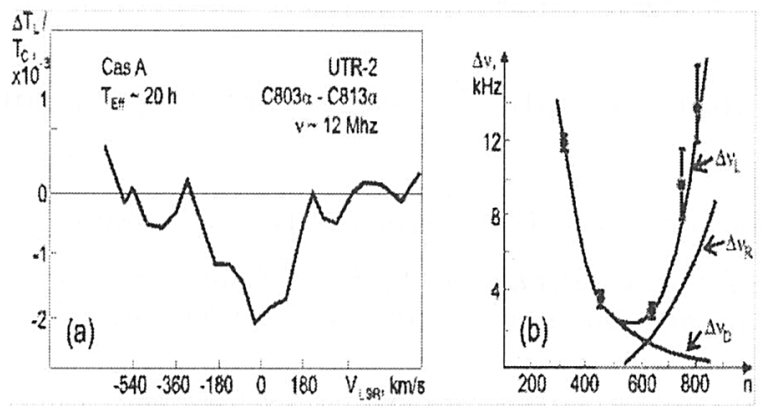

Figure 2. (a) - The spectra of the lowest frequency RRL; (b) - RRL broadening at very low frequencies.

Acknowledgments. The activity described here is supported in part by grants INTAS 96-0183, INTAS 97-1964.

\section{References}

Konovalenko, A. A. 1990, in Radio Recombination Lines: 25 Years of Investigations, eds. M. A. Gordon \& R. L. Sorochenko (Dordrecht: Kluwer), 178

Stepkin, S. V. 1996, Radio Physics \& Radio Astronomy (in Ukrainian), 1, 255

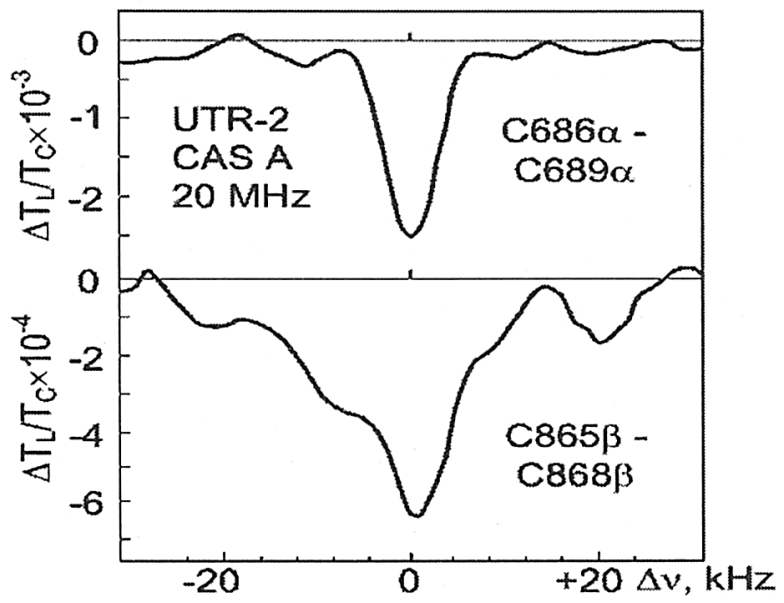

Figure 3. The detection of the highest principal quantum number of space atoms near $20 \mathrm{MHz}$. 